Weckx, S.; D'Hulst, R.; Driesen, J., "Primary and Secondary Frequency Support by a MultiAgent Demand Control System," Power Systems, IEEE Transactions on , vol.PP, no.99, pp.1,11

Digital Object Identifier: 10.1109/TPWRS.2014.2340582

URL: http://ieeexplore.ieee.org/stamp/stamp.jsp?tp=\&arnumber=6874591

IEEE. Personal use of this material is permitted. Permission from IEEE must be obtained for all other users, including reprinting/ republishing this material for advertising or promotional purposes, creating new collective works for resale or redistribution to servers or lists, or reuse of any copyrighted components of this work in other works. 


\title{
Primary and secondary frequency support by a multi-agent demand control system
}

\author{
Sam Weckx, Student Member, IEEE, Reinhilde D'Hulst, Johan Driesen, Senior Member, IEEE
}

\begin{abstract}
Decentralized demand control can help to ensure the balance between electricity demand and supply. In this paper, a multi-agent demand control system is proposed where residential demand is controlled to provide spinning reserves. With the proposed control framework an aggregator of dynamic demand is able to control the consumption and the response on frequency changes of a cluster of loads. The primary frequency support by the cluster of loads can emulate the primary control of a conventional generator. The total customer welfare remains maximal during the frequency support by applying utility functions for each device.
\end{abstract}

Index Terms-Demand response, Distributed optimization, Frequency control, Multi-agent control, Spinning reserves

\section{INTRODUCTION}

A high penetration of renewable energy sources challenges the future grid operation. In a power system the imbalance between generation and demand should be minimized as much as possible to ensure frequency stability and avoid blackouts. Renewable energy sources like solar and wind power are characterised by fluctuating power generation, while the amount of dispatchable power generation reduces. This makes it harder to match the production of energy to the demand. By controlling demand the more expensive alternative of flexible additional back-up generation can be avoided. The residential demand side can make a significant and reliable contribution to primary frequency response [1]. Different types of demand can be applied for frequency support, including fridges, freezers, HVAC and water heaters [1]-[3]. In [4] thermostatically controlled loads respond to the frequency by making their setpoints dynamic. The introduction of electric vehicles (EVs) in the electric network is another form of controllable load that can react on the frequency [5], [6]. It is shown in [7] that EVs can significantly reduce frequency deviations in isolated power systems.

Three levels of control are generally used to maintain the balance between supply and demand. Primary frequency control is a local automatic control that adjusts the active power generation and consumption to quickly restore the balance between load and generation [8]. Secondary frequency control is a centralized automatic control that adjusts the active power production or consumption to restore the frequency and the interchanges with other systems to their target values

S. Weckx is with ELECTA, KU Leuven, Kasteelpark Arenberg 10, bus 2445, 3001 Leuven, Belgium and with VITO, Boerentang 200 Mol, Belgium. He has a PhD fellowship of the Research Foundation Flanders (FWO-VITO). J. Driesen is with ELECTA, KU Leuven, Kasteelpark Arenberg 10, bus 2445, 3001 Leuven, Belgium.

R. D'Hulst is with VITO,Boerentang $200 \mathrm{Mol}$, Belgium. VITO and ELECTA are members of the Energyville research institute.

Corresponding author's email address: sam.weckx@esat.kuleuven.be following an imbalance [8]. Tertiary frequency control consists of redispatching the generating units [8]. The response time of each type of control is different. The primary frequency control reacts very quickly within the first few seconds after a disturbance. The secondary reserves can be activated in 30 $\mathrm{s}$ to $15 \mathrm{~min}$. Tertiary reserves are manually activated after the secondary reserves.

In literature there are still multiple issues concerning the application of dynamic loads as frequency reserves. First of all, the contribution of dynamic demand to primary and secondary reserves could be combined with a classical demand response system where the consumption of these loads is shifted through time. To be able to provide reserves, the load aggregator, that provides reserves by dynamic residential demand, needs to manage his cluster of loads so that the consumption can always be increased or decreased. The amount of reserves needs to be known by the aggregator and for primary frequency reserves the response of the cluster to frequency changes needs to be controlled.

In literature the power-frequency relationship of a device that participates in frequency control is not assumed to depend on the utility function of that device. A utility function describes the degree of well-being the product provides for consumers. Therefore, at an instance of frequency support by dynamic demand, social welfare is not maximized. An EV with a near departure time and a high requirement for sufficient energy will have a higher utility for power than an EV with an expected departure the next day. Therefore, when reducing demand to support the frequency, welfare maximization would result in decreasing the full charging power of the second EV while keeping the charging power of the EV with near departure time constant, rather than reducing both charging powers by an equal percentage. A similar analogy can be made for electric water heaters, fridges and freezers. The value of power for a fridge is indirectly incorporated by making the frequency-power relationship of the fridge dependent on temperature in [2]. In [9] the cost for providing a certain amount of symmetric primary reserves is minimized, where comfort constraints of the devices are taken into account to order the activation of binary behaving devices. However a more general tool to define the value of power for a certain device is their utility function. Given the utility of a device, social welfare can be maximized. In previous work [10] this concept was applied to primary frequency reserves.

In this paper a multi-agent control framework is presented that can shift demand through time and can activate primary and secondary reserves provided by a cluster of loads. The focus in this work is on the activation of frequency reserves, the reservation is outside the scope of this work. During 
activation of the reserves provided by dynamic demand, social welfare remains maximal. The paper is structured as follows: in section II, utility functions that describe the value of energy for different types of devices are presented. Section III introduces the market based multi-agent control used and shows how the multi-agent control framework activates contracted fast spinning reserves provided by the cluster of loads. In section IV a rolling horizon control is presented that determines the consumption plan of the cluster and that ensures the availability of the contracted reserves. Finally, section $\mathrm{V}$ discusses different simulation results of the proposed algorithm.

\section{UTILITY FUNCTION OF A DEVICE}

Energy can have a different value for each device depending on their state. This is generally expressed by a utility function, a concept applied in microeconomics. It should be strictly distinguished from the electric utility, which has another meaning. The utility function applied in microeconomics describes the degree of well-being the product provides for consumers. It therefore defines the different responses of different devices to various prices [11]. In this paper we assume utility functions that are decomposable in time and can be created for all types of devices. Popular utility functions include the linear and the quadratic utility function [11] denoted as $U\left(x_{i}\right)$, where $x_{i}$ is the power consumption level of the device $i$. A device $i$ that consumes $x_{i} \mathrm{~kW}$ electricity at a rate of $\lambda$ per $\mathrm{kWh}$ is charged $\lambda x_{i}$ per hour. Hence, the welfare of each user is defined as [11], [12]:

$$
W\left(x_{i}\right)=U\left(x_{i}\right)-\lambda x_{i}
$$

Where $W\left(x_{i}\right)$ is the users welfare function. Given a certain price $\lambda$, the power $x_{i}^{*}(\lambda)$ that maximizes the welfare of device $i$ is therefore defined as:

$$
x_{i}^{*}(\lambda)=\underset{x_{i}}{\operatorname{argmin}}-U\left(x_{i}\right)+\lambda x_{i}
$$

The function defined by $x_{i}^{*}(\lambda)$ is the optimal bid a device would make to an auction based market. When this bid is used, each price will result in maximum social welfare of the device. When the utility function is linear or quadratic, and the feasible sets are intervals, an explicit solution exists for $x_{i}^{*}(\lambda)$ [10], [13]. Devices that can only be turned on or off require a two-state utility function:

$$
U=\left\{\begin{aligned}
\beta_{i} & \text { if device is turned on } \\
0 & \text { if device is turned off }
\end{aligned}\right.
$$

Where the parameter $\beta_{i}$ characterizes the satisfaction if the device is turned on. The corresponding bid function is:

$$
x_{i}^{*}(\lambda)=\left\{\begin{aligned}
P_{i}^{\text {On } / O f f} & \text { if } \lambda \leq \frac{\beta_{i}}{P_{i}^{\text {On/Off }}} \\
0 & \text { if } \lambda>\frac{\beta_{i}}{P_{i}^{\text {On/Off }}}
\end{aligned}\right.
$$

Where $P^{O n / O f f}$ is the power consumption when the device is turned on.

A simple way of calculating a bid function for an EV, is by using a corner price $p_{r}$ [14], [15], as depicted in the left
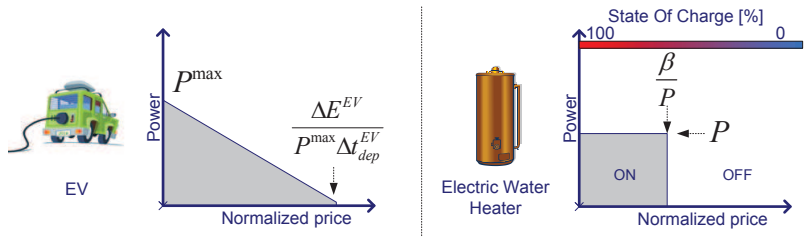

Fig. 1: Possible ways of defining a bid function $x_{i}^{*}(\lambda)$ for EVs and electric water heaters. The bid of an EV depends on the required energy by the time of departure and the time till departure. The bid of an electric water heater depends on the state of charge of the heater.

of Fig. 1. The corner price $p_{r}$ is the maximal price the EV is willing to pay for electric energy at that time instance and is represented by the following formula:

$$
p_{r}=\frac{\triangle E^{E V}}{P^{\max } \triangle t_{d e p}^{E V}}
$$

Where $\triangle E^{E V}$ is the required energy by the time of departure, $P^{\max }$ is the maximal charge power and $\triangle t_{d e p}^{E V}$ is the time till departure. EVs with high requirements for energy and little time left before departure will have higher bid functions. More on defining bid functions for EVs can be found in [14], [16].

Electric hot water heaters are other devices that offer a great amount of flexibility. Their charging can be shifted with little impact on the comfort of the user. It is assumed that the electric water heaters can not modulate their power consumption and have a fixed power consumption $P_{i}^{O n / O f f}$ when they are turned on. The State Of Charge (SOC) is the main indicator of a domestic hot water buffer for any demand response control system [17]. The closer the SOC drops to its minimum, the more urgent its scheduling. Therefore the bid is made inversely proportional to the SOC of the heater. This is done by making $\beta_{i}$ of (3) and (4) inversely proportional to the SOC. In case of a low SOC, the bid will be high and vice versa, as depicted in the right of Fig. 1.

A similar reasoning holds for other devices [10]. Note that the optimal bidding is not limited to these types of bids, but can incorporate other factors as well, like consumer preferences or consumer wealth. The utility functions are not limited to the described functions, but are assumed to be decomposable in time and to be non-decreasing. The class of utility functions that fulfil these conditions is very large [11], [18]. When devices compose their own utility function, the assumption that consumers are very sophisticated, to the level of being daily energy traders that are aware of their utility functions, can be avoided. In this paper EVs and electric water heaters are used as they can instantly adapt their power consumption.

\section{Auction BASED CONTROL}

A scalable auction based control framework is applied to control the cluster of loads. It is extended to activate the contracted spinning reserves. The multi-agent control algorithm 


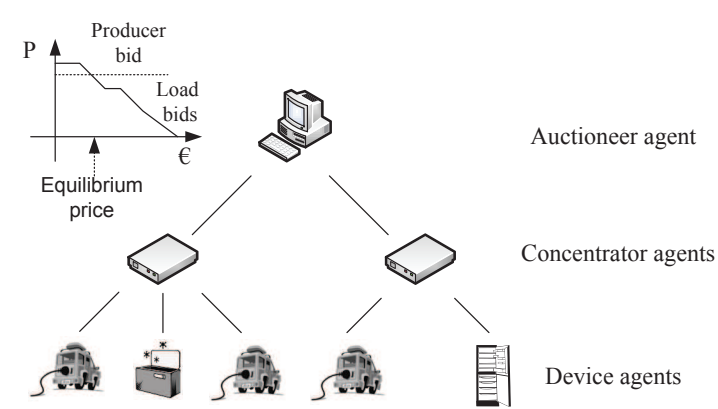

Fig. 2: Auction based power market.

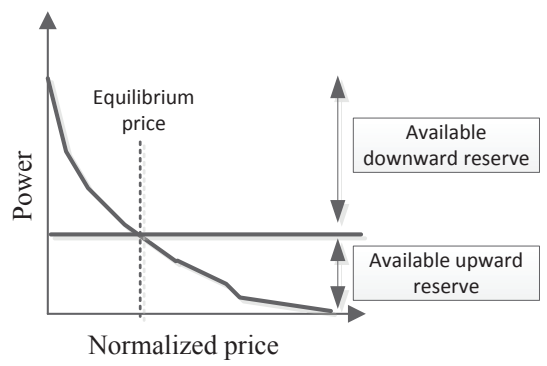

Fig. 3: Available reserves of the cluster of loads after defining the equilibrium price.

consists of two parts: one part to control the cluster of loads in real-time to obtain the planned consumption and another part for the planning of the consumption of the cluster of loads over a time horizon. The former is covered in this section, while the latter is discussed in section IV.

\section{A. Real-time control of the loads}

In the real-time operation of the multi-agent algorithm all the device agents send their bid $x_{i}^{*}(\lambda)$ to a concentrator agent. This concentrator agent sums up the bid functions of their zone [19], [20]. The concentrator agents in turn send the bid function to a unique auctioneer agent. Finally, the auctioneer agent will define the equilibrium price as the intersection of the aggregated bid functions and the supply bid function. The supply bid function is defined by the consumption level $P_{t}^{\text {Load }}$ that was planned by the auctioneer agent. How to define this consumption level $P_{t}^{\text {Load }}$ will be discussed in section IV. The auctioneer agent manages and plans the real-time consumption of the cluster of loads of the load aggregator. After the equilibrium price is defined, it is sent back to all of the device agents and these will then select their corresponding power level. This market clearing takes place every 15 minutes or can be made event-driven. Fig. 2 presents this auction based market approach.

When consumption can be increased or decreased given the defined equilibrium price, the cluster of loads is able to provide upward and downward reserves. This is shown in Fig. 3. This multi-agent control framework can be extended to cooperate in frequency reserve markets. For the cluster of loads to be activated as reserves, extensions have to be made to the part of the multi-agent system responsible for the real-time operation.
The provided frequency reserves are divided into primary or Frequency Containment Reserves (FCR) and secondary or Frequency Restoration Reserves (FRR). The purpose of this work is to imitate these reserves provided by conventional generators with a cluster of loads. Primary frequency reserves by conventional generators respond instantaneously to frequency changes. To mimic this behaviour, the instantaneous primary frequency control by dynamic demand cannot be based on any form of communication. Therefore a local controller taking care of the primary frequency control is added to each device agent. In case of a frequency drop, the power consumption that creates the least welfare should be shut down. In case of a frequency rise, the power consumption that results in the highest extra welfare should be activated. The response of the local controller can be changed and optimized by the auctioneer agent. Secondary reserves should be available between $30 \mathrm{~s}$ and 15 min after a request of the transmission system operator and therefore a reliable communication system can be applied to activate the required reserves by the dynamic demand. For ease of explanation the secondary frequency control will be discussed before the primary frequency control by dynamic demand.

\section{B. Secondary frequency control}

Due to the slower time scale of secondary control the auction based infrastructure, as described in section III-A, can be applied to activate secondary reserves. The total consumption of the cluster of loads $\sum_{i=1}^{N} x_{i}$ needs to be equal to the sum of the planned load $P_{t}^{L \text { Load }}$ of the cluster at time step $t$ and the activated upward or downward reserves $R_{\text {load }}^{2,+/-}$. To obtain this consumption the auctioneer agent makes the supply bid function equal to $P_{t}^{\text {Load }}+R_{\text {load }}^{2,+/}$. The resulting equilibrium price gives rise to the required consumption level of the cluster of loads when sent to all devices.

The secondary frequency control with the multi-agent auction based control is a distributed utility maximization problem. The utility maximization problem equals:

$$
\begin{array}{ll}
\max _{x} & \sum_{i=1}^{N} U\left(x_{i}\right) \\
\text { subject to } & \sum_{i=1}^{N} x_{i}=P_{t}^{\text {Load }}+R_{\text {load }}^{2,+/-}
\end{array}
$$

The dual of this problem is defined as:

$$
\max _{\lambda} \min _{x}-\sum_{i=1}^{N} U\left(x_{i}\right)+\lambda_{m}\left(\sum_{i=1}^{N} x_{i}-P_{t}^{\text {Load }}-R_{\text {load }}^{2,+/-}\right)
$$

If strong duality holds then the solution of the dual problem (7) equals the solution of the primal problem (6). For integer problems, strong duality in general does not hold. In [21] is stated that problems of this type have a diminishing duality gap if the number of subproblems increases. Therefore solving the dual problem with a high amount of aggregated devices will result in only a small or even no error. 
The Lagrangian dual function is decomposable in different subproblems. The welfare maximization that each device performs in (2) is a subproblem of the dual problem. It can be shown that $\sum_{i=1}^{N} x_{i}^{*}\left(\lambda_{m}\right)-P_{t}^{\text {Load }}-R_{\text {load }}^{2,+/-}$ is a subgradient of the dual problem and therefore finding the intersection of the aggregated bid functions $\sum_{i=1}^{N} x_{i}^{*}\left(\lambda_{m}\right)$ and the supply bid function $P_{t}^{\text {Load }}+R_{\text {load }}^{2,+/-}$ comes down to solving the dual problem of the utility maximization problem. The equilibrium price is equal to the lagrange multiplier $\lambda_{m}$ of the dual problem.

Intuitively, when all customers receive the same price and this price results in the desired consumption level of $P_{t}^{\text {Load }}+$ $R_{\text {load }}^{2,+/-}$, the total utility is maximized, as only devices with a sufficient need for energy are turned on.

\section{Primary frequency control}

Primary frequency reserves are used to stabilise the frequency after a disturbance in the time frame of seconds. Conventional generators react immediately to this disturbance. To mimic the behaviour of these conventional generators, the response of the loads can therefore not depend on a communication signal that is sent after the occurrence of such a disturbance. Instead it is based on a local controller that is added to the device agent.

When the frequency is higher than the nominal value an incentive for consumption should be given, when it is lower, consumption should be decreased. This can be achieved by adding an extra frequency dependent price component to the normal price. The market clearing price that customers receive is the price at the nominal frequency, i.e. $50 \mathrm{~Hz}$, which results in a consumption level of $P_{t}^{\text {Load }}+R_{\text {load }}^{2,+/-}$. A frequency deviation results in a price deviation from that market clearing price. The auctioneer therefore does not send only one equilibrium price to all loads, but a price dependent on frequency. The frequency dependent price is defined by the preferred shape of the frequency response of the cluster of loads. Fig. 4 shows the aggregated bid function and the resulting power-frequency droop relationship. The dotted line is the price at $50 \mathrm{~Hz}$. In a deadband around $50 \mathrm{~Hz}$, the price remains unchanged. When the frequency deviates outside the deadband, devices adapt their behaviour as defined by their bid functions. The local control parameters of a device then depend on the bid functions of the device as described in section III. The bid function defines the power-price relationship and therefore also defines the power-frequency relationship. An example of two EVs is given in Fig. 5. The EV with a near departure time and little energy stored in the battery is charging and only stops charging when the frequency significantly drops. The EV with a larger amount of time left before departure only starts charging when the frequency reaches a certain value above $50 \mathrm{~Hz}$.

The aggregated response of all loads mimics the behaviour of a conventional generator. When more devices are involved in the auction based control the aggregated bid function

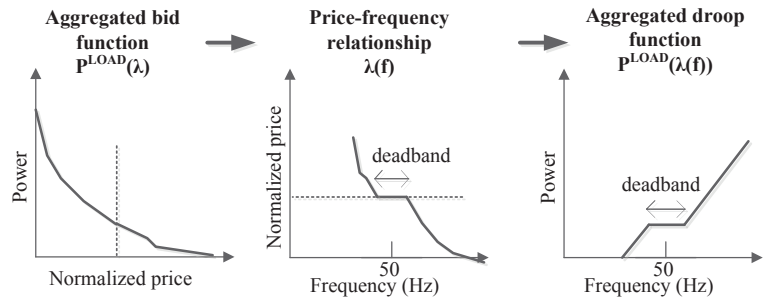

Fig. 4: To obtain a specific power-frequency or droop relationship of the cluster of loads the price is determined as a function of the frequency. The dotted line is the market price at $50 \mathrm{~Hz}$.

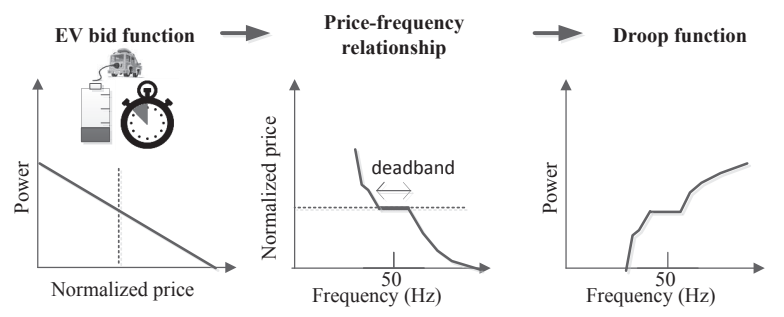

(a) The derived frequency droop function for an EV with a high and urgent need for energy.

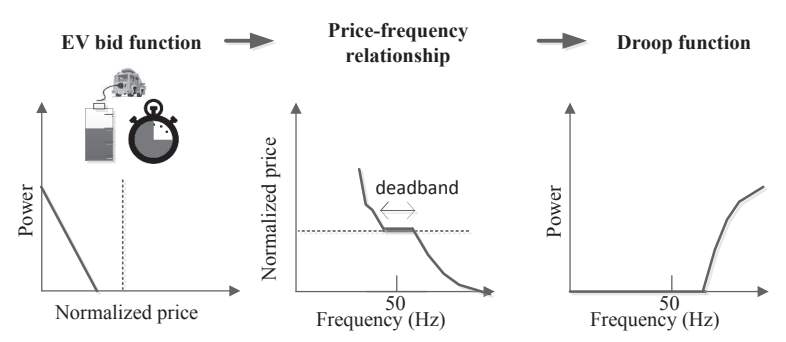

(b) The derived frequency droop function for an EV with a low need for energy.

Fig. 5: The individual droop relationship of each device depends on their bid function. The price-frequency relationship is always equal for all loads of the cluster. The dotted line is the market price at $50 \mathrm{~Hz}$.

becomes smoother and therefore the smoother behaviour of the conventional generators can be imitated. The primary frequency control can be made both symmetric and asymmetric.

Devices can autonomously adapt their power consumption based on the frequency-price dependency. This results in consumption levels that are dependent on frequency, while the system welfare remains maximal. As frequency can be measured locally in a cheap way [2], devices can adapt their own price automatically. The devices do not require any information about the system, in contrast to the method 

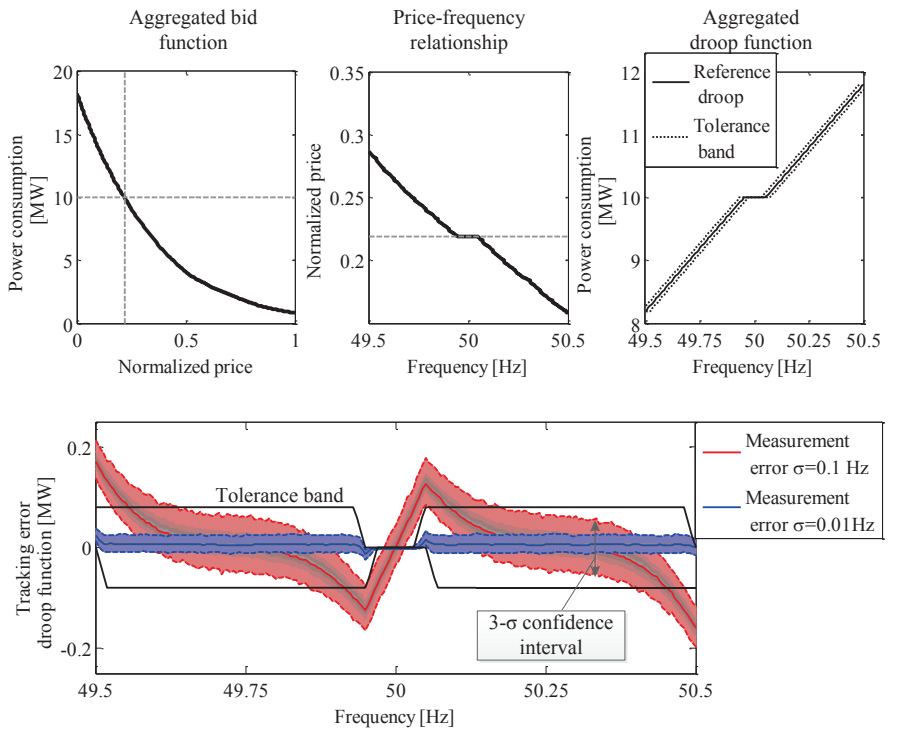

Fig. 6: Influence of frequency measurement errors on the aggregated droop function.

described in [22]. The only difference with the classic auction based control is that instead of one single equilibrium price, a frequency-price relationship is communicated to all loads. The frequency-price relationship is always equal for all controllable loads. At time steps that loads are not used for primary reserves, the price will be independent of the frequency.

Frequency can be considered to be equal across the system and therefore the price increase will be equal for all customers, which will result in maximal system utility. Only small errors on the frequency measurements give rise to minor price differences that could deteriorate the maximal system utility. The effect of frequency measurement errors on the aggregated droop function is plotted in Fig. 6. $5000 \mathrm{EVs}$ and 5000 electric water heaters make a bid. At the top of this figure the aggregated bid function is shown. The price-frequency relationship that results in the aggregated droop function is also shown. A controller tolerance band of $\pm f_{t o l}=20 \mathrm{mHz}$ is added to the aggregated droop function [9]. Frequency measurement errors result in a deviation of the reference droop function and can result in difficulties to comply with an acceptable tolerance band. Uncorrelated Gaussian noise is added to the frequency measurements of the participating devices. Gaussian noise with a $\sigma$ equal to $0.01 \mathrm{~Hz}$ results in a minimal deviation from the reference droop curve and compliance with the tolerance band. A $\sigma$ equal to $0.1 \mathrm{~Hz}$ results in a severe deviation of the reference curve, which does not comply with the accepted tolerance band as shown in the bottom part of Fig. 6. It is therefore recommended to reduce the frequency measurement errors to below the tolerance band.

The primary frequency support activation has a distributed optimization interpretation. The purpose of the primary load frequency support by dynamic demand is to eliminate the loadgeneration mismatch in the whole system, while maximizing system utility. Intuitively if customers receive the same price and this price converges, the system utility will remain maxi-

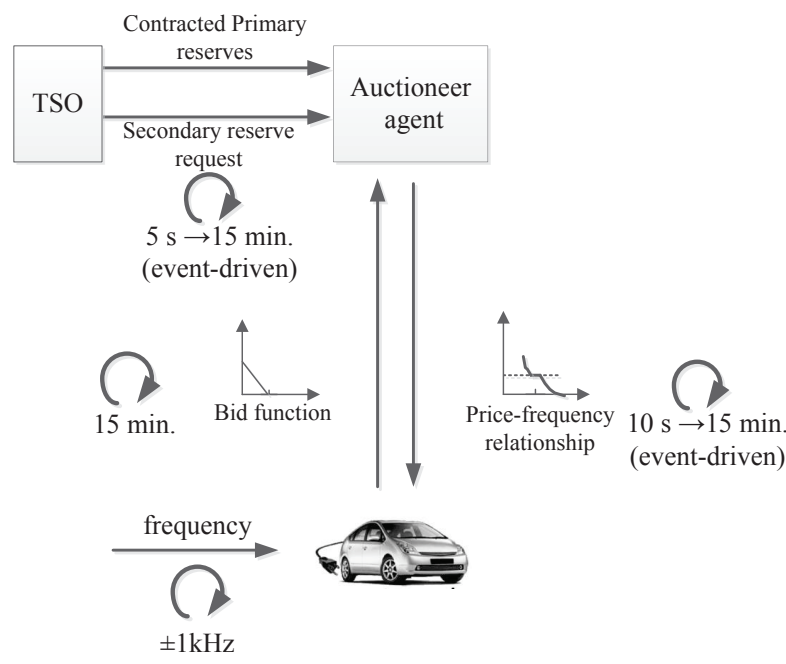

Fig. 7: The interaction between the different agents. The concentrator agent is not presented.

mal. A mathematical proof can be found in the appendix A.

\section{Practical considerations}

The exchange of information between the different parties is presented in Fig. 7. The secondary control actions $R_{\text {load }}^{2,+/-}$ are usually determined at a central dispatch centre based on the Area Control Error (ACE) [23]. The control signals of the dispatch center are transmitted to the generating units and to the auctioneer agent. The auctioneer agent can adapt the pricefrequency relation to comply with the requested action. The maximum amount of time that can elapse between the request from the TSO and the beginning of the response by the loads depends on the country and can range from a few seconds to 5 minutes [8]. Note that this system is perfectly suited for an event-driven implementation [24] where the price-frequency relationship gets updated after each request of the system operator.

The device agent locally measures the frequency, and responds to the frequency according to the last received pricefrequency relationship. Therefore the devices can comply with the timing requirements of the primary frequency reserves. The device agent updates his bid function every 15 minutes, to reflect its actual value for the available energy.

It is assumed that the customers are not charged the frequency-dependent price. In the auction based market control of [16], [24], [25] the customers are also not charged the equilibrium price. Customers offering flexibility can be reimbursed for the offered flexibility, for the used flexibility or by a yearly fixed fee. The frequency-dependent price can therefore be interpreted as a control signal. This control signal guarantees that the necessary reserves are provided, while the total welfare of all devices remains maximal. The algorithm remains identical when the equilibrium price would be charged to the customer. One of the main critics in these 
types of algorithms is the assumption that consumers are very sophisticated, to the level of being daily energy traders that are aware of their utility functions. When the price is not charged and the devices compose their utility function based on the heuristics described in section II, this assumption is omitted.

\section{REAL-TIME PLANNING OF THE CLUSTER CONSUMPTION}

A load aggregator will try to use the available flexibility to maximize its profits during a whole day. It is therefore required to take into account future information when the actual cluster consumption is defined by the auctioneer agent. This is done by a rolling horizon control as described in [16], [24]-[27]. Further extensions are required to these frameworks to guarantee the availability of the contracted reserves.

This work focusses on the real-time decisions made concerning the real-time consumption of the loads and the activation of the contracted reserves. The day-ahead decisions are outside the scope of this work. The contracted primary $R_{\text {load } t}^{1,+/}$ and secondary $R_{\text {load }, t}^{2,+/}$ reserve capacity and the planned dayahead consumption $P_{t}^{D A}$ are therefore assumed to be known, while the real-time consumption levels $P_{t}^{\text {Load }}$ are optimized. Note that the actual cluster consumption can differ from the planned real-time consumption $P_{t}^{\text {Load }}$ due to the activation of the contracted reserves.

The determination of a collective consumption plan for a cluster of aggregated loads is extensively discussed in [16], [24]-[26]. However it will require several extensions to incorporate the activation of fast spinning reserves.

The objective of the load aggregator is to maximize his profits for the given period by making a power consumption plan that respects the device limitations. The load aggregator is active on the real-time imbalance market. When the real-time consumption does not match the planned day-ahead consumption, an imbalance price is used by the TSO to calculate the reimbursement or penalty the aggregator receives. Activated reserves are not included in the calculation of the aggregator his imbalance. To increase his profits, the aggregator can consume less than the planned day-ahead consumption during a high positive imbalance price to receive a high reimbursement. The extra energy can be consumed during a low negative imbalance price which will result in a small penalty. I.e. during an unexpected excess of wind power, the negative imbalance price can become zero. The objective of the aggregator that maximizes his profits on the imbalance market equals ${ }^{1}$ :

$$
\begin{array}{r}
\max _{P_{t}^{\text {Load }}} \quad \sum_{t=1}^{T} c_{i m b, t}^{+}\left[P_{t}^{D A}-P_{t}^{\text {Load }}\right]_{+} \Delta t+\ldots \\
\ldots-c_{i m b, t}^{-}\left[P_{t}^{\text {Load }}-P_{t}^{D A}\right]_{+} \triangle t
\end{array}
$$

where $c_{i m b, t}^{-}$and $c_{i m b, t}^{+}$are the expected negative and positive real-time imbalance prices. The deviation is the difference between the day-ahead contract $P_{t}^{D A}$ and real-time power demand $P_{t}^{\text {Load }}$ at time step $t$. During each time period the maximum consumption of the cluster is limited. The

\footnotetext{
${ }^{1}$ For $\mathrm{a} \in \mathbb{R},[\mathrm{a}]+$ denotes the $\max [\mathrm{a}, 0]$
}

auctioneer agent makes an estimation of the minimum $\left(P_{t}^{\min }\right)$ and maximum power $\left(P_{t}^{\max }\right)$ the cluster of loads can consume each time step. Due to the repetitive behaviour of customers these parameters can be estimated. The cluster of loads also has a limited amount of energy consumption. These limitations can be described by the flexibility bounds of the cluster $E^{\max }$ and $E^{\min } . E^{\max }$ is defined as the accumulated energy if all loads were to start immediate consumption at maximum power and then idle, while $E^{\text {min }}$ is when consumption is postponed by all loads as long as possible. The EVs and domestic water heaters send their individual flexibility bounds to the auctioneer agent [16], [24]-[26]. This way the auctioneer has historic information of daily flexibility bounds. The constraints describing the cluster limitations are therefore:

$$
\begin{aligned}
P_{t}^{\text {min }} & \leq P_{t}^{\text {Load }} \leq P_{t}^{\text {max }} \\
E_{t+1} & =E_{t}+P_{t}^{\text {Load }} \triangle t \\
E_{t}^{\text {min }} & \leq E_{t} \leq E_{t}^{\text {max }}
\end{aligned}
$$

The previous control formulation as described in [16], [24][26] does not incorporate the possible activation of contracted reserves. As was shown in Fig. 3, to be able to provide upward reserves, the cluster needs to consume a minimal amount of power, whereas downward reserves require the cluster to not consume the maximal amount of power. Besides that, when one MW is already contracted as a primary reserve capacity, it cannot be procured again as a secondary reserve. The consumption of the cluster $P_{t}^{\text {Load }}$ is therefore bound by:

$$
\begin{aligned}
& P_{t}^{\text {min }} \leq P_{t}^{\text {Load }}-R_{\text {load }, t}^{1,+}-R_{\text {load }, t}^{2,+} \\
& P_{t}^{\text {Load }}+R_{\text {load }, t}^{1,-}+R_{\text {load }, t}^{2,-} \leq P_{t}^{\text {max }}
\end{aligned}
$$

The amount of primary and secondary reserves that will be activated during the next time step is unknown. The sum of $R_{\text {load }, t}^{1, \pm}+R_{\text {load }, t}^{2, \pm}$ is therefore chosen to be equal to the amount of contracted reserves to guarantee their availability. The aggregated cumulated energy of the cluster depends on the activation of the reserves provided by the cluster and can be represented by two scenarios:

$$
\begin{aligned}
& E_{t+1}^{+}=E_{t}^{+}+\left(P_{t}^{\text {Load }}-R_{\text {load }, t}^{1,+}-R_{\text {load }, t}^{2,+}\right) \Delta t \\
& E_{t+1}^{-}=E_{t}^{-}+\left(P_{t}^{\text {Load }}+R_{\text {load }, t}^{1,-}+R_{\text {load }, t}^{2,-}\right) \Delta t
\end{aligned}
$$

When no reserves are activated, the cumulated energy is noted as $E_{t+1}$. If all contracted upward reserves would be activated during the day the cumulated energy would equal $E_{t+1}^{+}$and when all contracted downward reserves would be activated the cumulated energy would equal $E_{t+1}^{-}$. These last two are the worst-case scenarios, whereas $E_{t+1}$ can be considered as the expected scenario. For all possible scenarios, the cumulated energy is limited by the flexibility bounds of the cluster. This can be defined as:

$$
\begin{aligned}
& E_{t}^{\text {min }} \leq E_{t}^{+} \\
& E_{t}^{-} \leq E_{t}^{\text {max }}
\end{aligned}
$$

Each quarter hour the real-time power demand $P_{t}^{\text {Load }}$ is recalculated to adapt to the new estimates of the cluster flexibility and the new predictions of the imbalance prices. 

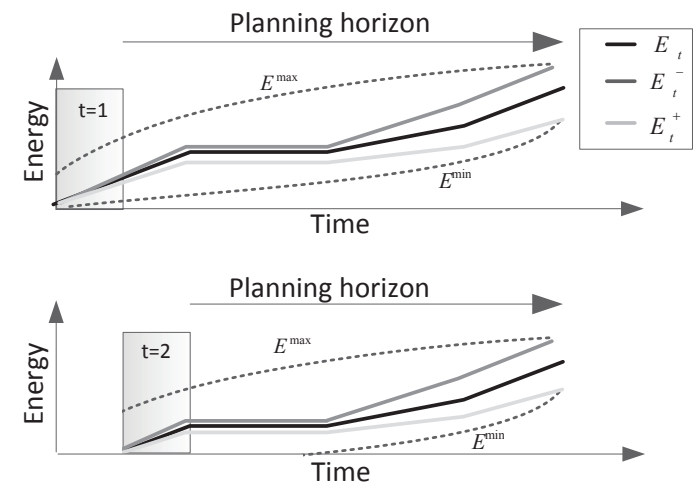

Fig. 8: Rolling horizon cluster load control.

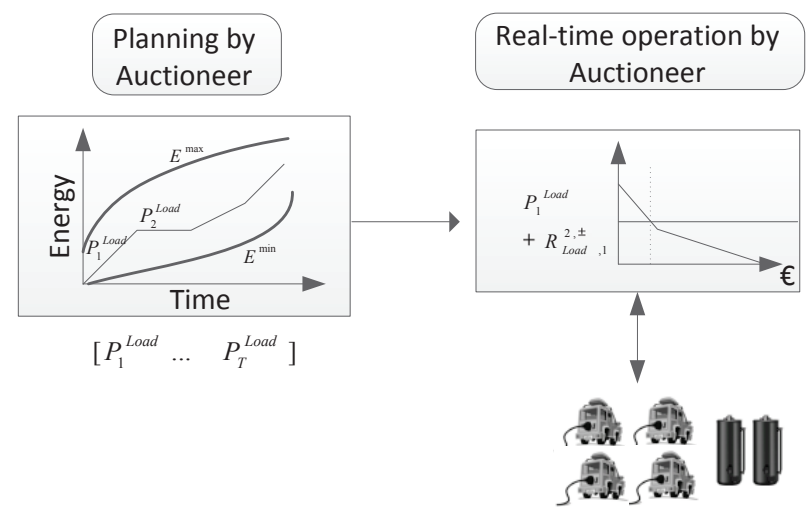

Fig. 9: Planning the power consumption by the cluster of loads.

Besides uncertainties on the cluster flexibility, the actual power consumption in the previous time step could be lower or higher due to the activation of reserves. This rolling horizon approach is presented in Fig. 8. Imbalance prices are assumed to be known in this work. To cope with the uncertainties regarding the predictions of the real-time imbalance prices, stochastic [28]-[30] or robust optimization techniques [28], [31] can be applied. In a stochastic optimization formulation different possible price scenarios are taken into account, while in a robust optimization formulation the price uncertainties will be described by uncertainty intervals. Including uncertainty on the real-time imbalance prices is outside the scope of this work.

By applying the heuristic of Fig. 2, the planned energy consumption for the actual time slot is distributed over the cluster of loads. As discussed in section III, if secondary reserves are requested $R_{\text {load, }}^{2, \pm}$, these should be added to the planned $P_{t}^{L o a d}$. If primary reserves are contracted, the frequency-dependent price is formed. This control approach is presented in Fig. 9. One of the main advantages of this approach is its scalability: the computation of the path planning is independent of the total amount of loads.

\section{Simulation Results}

The proposed primary frequency method is tested on the test grid presented in Fig. 10. In this grid there is a generating unit with a reheat steam turbine and a governor with droop action

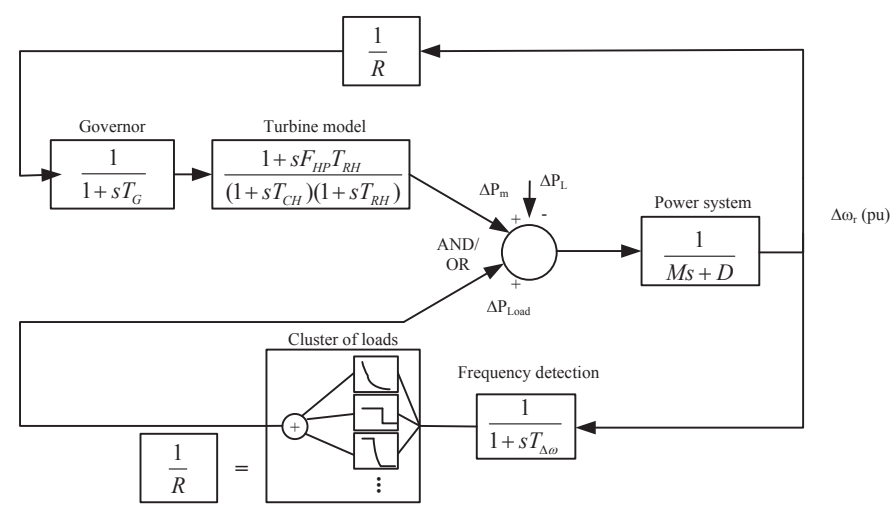

Fig. 10: Block diagram of the simulated system.

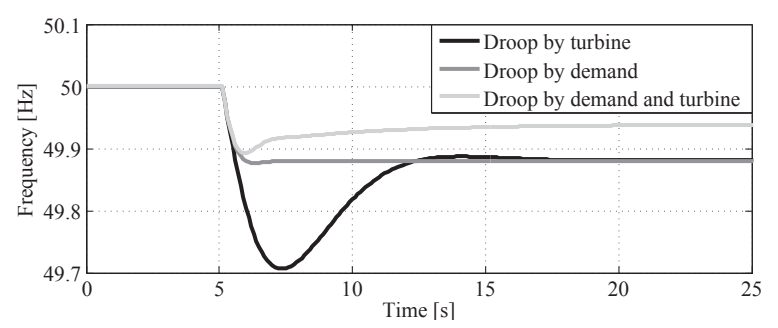

Fig. 11: Frequency transient when $5 \%$ of generation is lost.

as defined in [23]. The turbine time constants are $T_{R H}, T_{C H}$ and $F_{H P} T_{R H}$, the governor time constant is $T_{G}$ with a gain defined by $R$. $M$ is double the inertia constant $H$ and $D$ is the load-damping constant. Typical values of these constants can be found in [23]. The droop action by the reheat turbine can be turned off and replaced by a droop provided by the cluster of loads. Frequency detection by the devices is assumed to have a time constant $T_{\triangle \omega}$ equal to $0.2 \mathrm{~s}$ [32]. It is assumed that a device can change its consumption level with a time constant lower than $T_{\triangle \omega}$ which is therefore not modelled. Note that the aggregator could also send out a time constant bigger than $0.2 \mathrm{~s}$ to all devices to create a requested first order feedback loop. The droop constant of the reheat turbine is identical to the emulated droop constant by the cluster of loads and equals 0.05. Both types of control are compared in Fig. 11 where the system response is plotted when 5\% of generation is lost. The steady-state frequency is identical due to the identical droop constant, but the transient response differs. The cluster of loads has a fast response to the disturbance and therefore the frequency dip is less severe.

Fig. 12 plots the aggregated bid function, the applied pricefrequency relationship and the price evolution during this simulation. Reserves were contracted from $49.8 \mathrm{~Hz}$ to $50.2 \mathrm{~Hz}$, without a deadzone and an identical droop as the reheat steam turbine. In case the controllable demand is responsible for the primary frequency control, the price quickly increases to adapt the consumption of the controllable demand to the generation. The users have received the price-frequency relationship from the auctioneer agent, so there is no need to communicate this price in real-time. The users know the price based on 

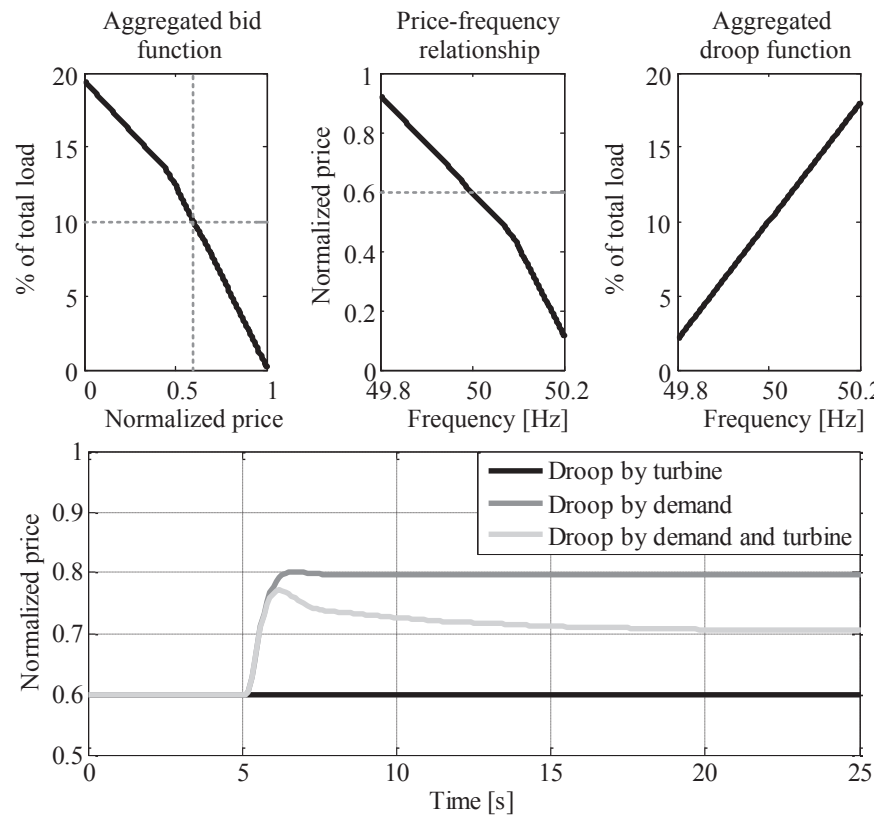

Fig. 12: The evolution of the price signal of the users when $5 \%$ of generation is lost.

their frequency measurement. When both the turbine and the controllable demand take part in the frequency control, it can be seen that the price quickly rises, but starts to decrease after a few seconds. The turbine has a slower response, so it will take a few seconds before the turbine provides his share in the primary frequency control. Due to the fast response of the devices, the frequency drop is reduced. When the controllable demand is not used for frequency reserves, the price is independent of the frequency and remains constant during the frequency drop.

Note from Fig. 12 that replacing the only conventional generator responsible for primary frequency control completely by controllable demand would require a high amount of controllable demand. Therefore the controllable demand will rather be just a part of the total primary reserves, where the fast response of the demand can reduce the frequency drop before slower conventional generators will help to restore the balance between generation and demand.

In a second simulation the real-time planning of the cluster of loads is evaluated. $5000 \mathrm{EVs}$ and 5000 electric water heaters are aggregated and are controlled by the multi-agent control framework. The maximum charging rate of the EVs is $3.3 \mathrm{~kW}$. Their availability and energy consumption are modelled based on [33]. The electric domestic water heaters have a capacity of 2001 and a maximum power consumption level of $3.3 \mathrm{~kW}$. Consumption data of the electric domestic water heaters was available from the LINEAR project [34]. The EVs make a linear bid to the auction based market, whereas the heaters make a block bid. Each quarter hour these bids are updated according to the state of the device. Upward and downward reserves of $0.5 \mathrm{MW}$ are contracted during the whole day, except from 10 AM to 4 PM. The Belgian imbalance prices of October 1st 2013 are applied, while the assumed activated reserves are based on the net regulation volume of that day. In Belgium the negative and positive imbalance prices $c_{i m b, t}^{-}$ and $c_{i m b, t}^{+}$are often almost identical.

The determination of the collective consumption plan for the cluster of loads during the day is done with the rolling horizon control framework described in section IV. Two consumption controllers are compared in Fig. 13: one takes into account the extra constraints (12-17) to guarantee the availability of the contracted reserves, while the other controller equals the one of [16], [24] where reserve constraints are neglected. To maximize profits, both controllers try to generate a lower consumption than planned during high imbalance prices, while consumption is increased during low imbalance prices. The controller that neglects the reserve constraints can reduce the consumption to lower values since it does not include a margin to provide upward reserves. Therefore it often fails to provide the reserves requested by the system operator. When comparing the accumulated energy paths of both control approaches, it is clear that the controller that takes into account the reserve constraints operates less closely to the flexibility bounds of the cluster and is therefore able to provide the contracted reserves. The day-ahead planning for both consumption controllers was obtained by the method described in appendix B with the dayahead prices of October 1st 2013.

\section{CONCLUSION}

A general multi-agent framework based on utility maximization is proposed for frequency support by dynamic demand. The multi-agent controller consists of two parts. One part is responsible for the real-time operation, while the other part is responsible for a real-time planning of the consumption plan. It was shown that when the algorithm activates primary and secondary reserves, the customer welfare remains maximal. Devices can identify their value of the received energy by making use of utility functions. With the proposed control framework the primary and secondary frequency control by a conventional generator can be imitated. A rolling horizon control is applied to define a consumption plan that maximizes the load aggregators profits. The controller takes into account the contracted spinning reserves to guarantee their availability in the future time periods.

\section{APPENDIX A}

\section{PRIMARY FREQUENCY SUPPORT}

The primary frequency support has a distributed optimization interpretation. The purpose of the primary load frequency support by dynamic demand is to eliminate the load-generation mismatch in the whole system, while maximizing system utility. This can be formulated as the following optimization problem:

$$
\min _{x}-\sum_{i=1}^{N} U\left(x_{i}\right)+\lambda_{m} x_{i}
$$

subject to

$$
\sum_{i=1}^{N} x_{i}+P_{\text {inel }}=G
$$




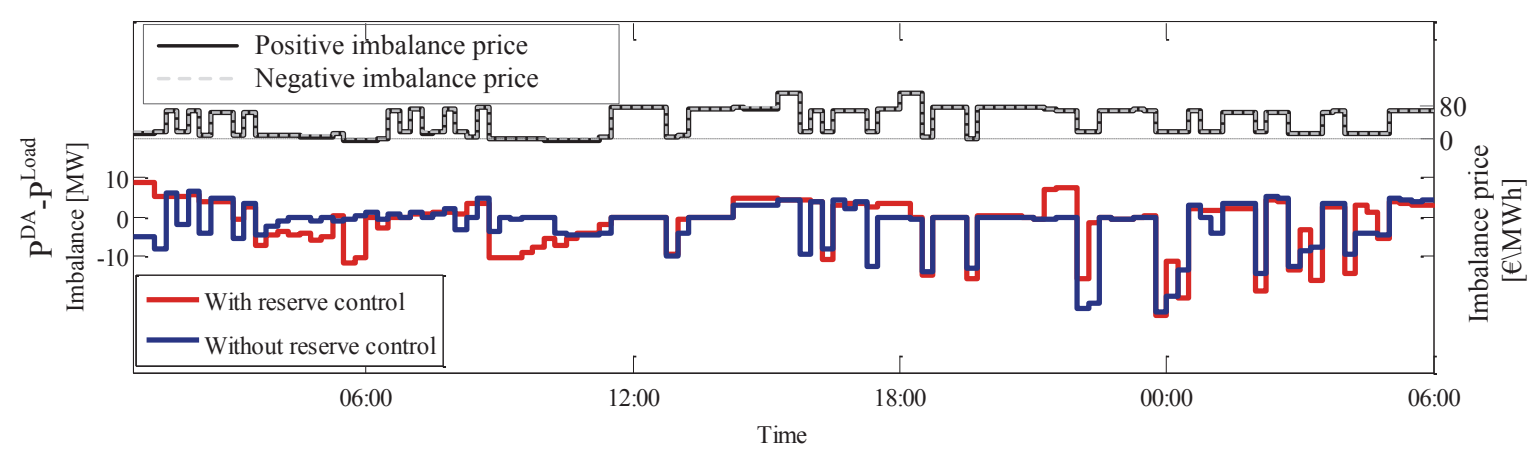

(a) To increase profits, the cluster of loads consumes less than the planned day-ahead profile during a high positive imbalance price and consumes more energy during a low negative imbalance price.

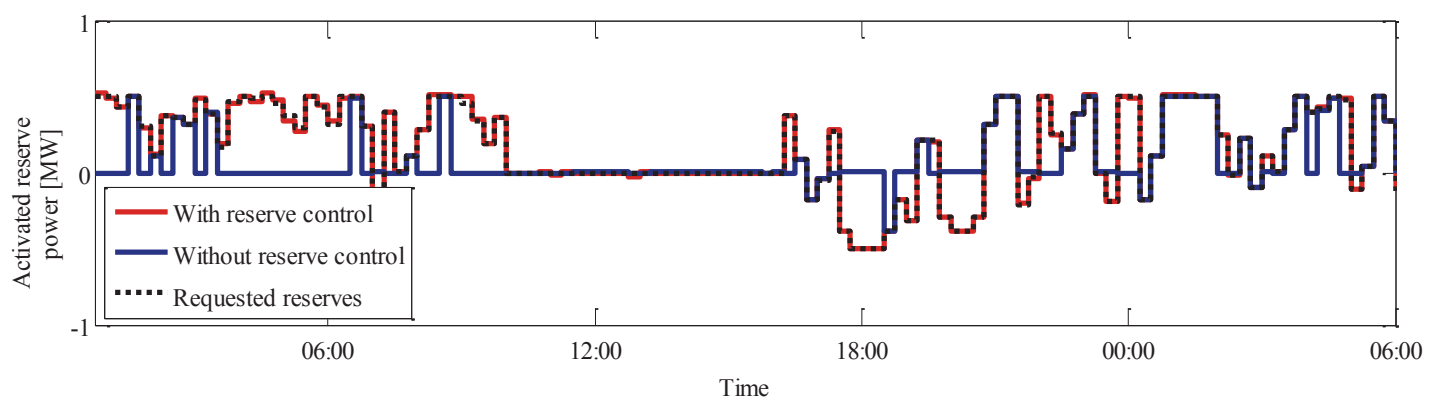

(b) The activated reserves and the tracking of these reserves are plotted. The reserves can often not be provided when they are not taken into account in the optimization formulation.

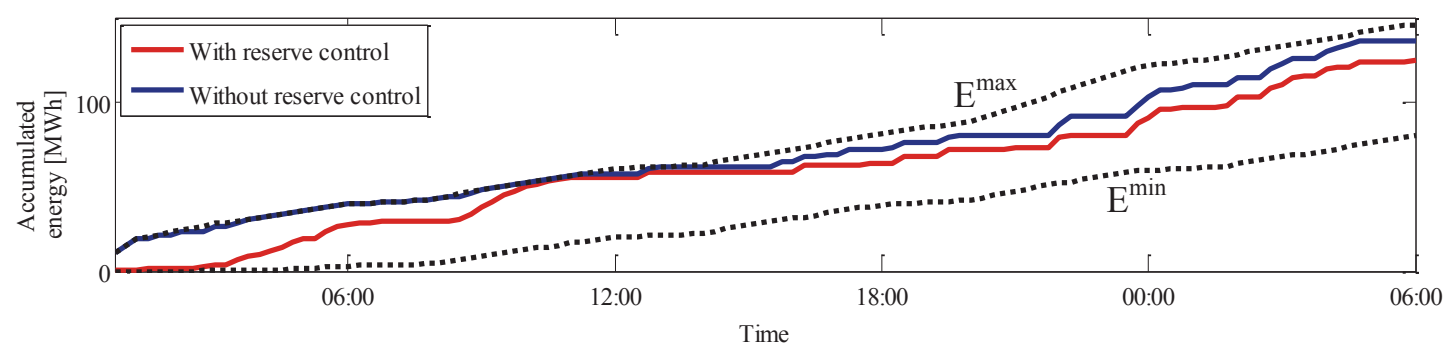

(c) The accumulated energy of the cluster of loads is plotted. The accumulated energy remains further from the flexibility bounds of the cluster of loads when reserves are taken into account. The contracted reserves can then always be provided in future time slots.

Fig. 13: A simulation of one day of the real-time planning of the cluster of loads with and without reserve control.

where $P_{\text {inel }}$ is the total load in the system except the $N$ devices participating in the frequency support and $G$ is the total generated power. $\lambda_{m}$ is the fixed market price for power at $50 \mathrm{~Hz}$. The Lagrangian dual function of this problem is defined as:

$$
\begin{aligned}
\Lambda\left(\lambda_{\text {freq }}\right)=\min _{x} . & -\sum_{i=1}^{N} U\left(x_{i}\right)+\lambda_{m} x_{i} \\
& +\lambda_{\text {freq }}\left(\sum_{i=1}^{N} x_{i}+P_{\text {inel }}-G\right)
\end{aligned}
$$

The maximization of the Lagrangian dual function is the dual problem. The dual problem can be solved by a dual ascent procedure [22] to find the optimal lagrange multiplier $\lambda_{f r e q}^{*}$ of the dual problem, which is the price that would eliminate the supply-demand imbalance. This method however requires that each device knows the system transfer function to estimate the supply-demand imbalance [22]. This is omitted in our work. The optimal lagrange multiplier $\lambda_{\text {freq }}^{*}$ will be found by the frequency dependent pricing described in section III-C.

The supply-demand imbalance $\left(\sum_{i=1}^{N} x_{i}+P_{\text {inel }}-G\right)$ is a subgradient of the dual problem. If this subgradient is zero, the concave unconstrained dual problem is solved.

The frequency deviation $\frac{\Delta \omega^{k}-\Delta \omega^{k-1}}{\Delta t}$ is a measure of the supply-demand imbalance. When the frequency deviation converges to zero, the supply-demand imbalance is zero. A stable frequency can only be obtained when the supply-demand imbalance is zero, as otherwise an excess or lack of energy in the system would increase or decrease the speed of the generators. So a stable frequency implies a supply-demand imbalance of zero and therefore the subgradient of the dual problem is zero. Therefore if the frequency dependent price of 
section III-C results in a stable power system, the frequency deviation will become zero and the concave unconstrained dual problem is solved. Customers respond to a price signal, independent of the way the price is obtained, so the price that results in a supply-demand imbalance of zero obtained with the dual ascent method of [22] is identical to the frequency dependent price that results in a supply-demand imbalance of zero obtained by the method of section III-C. I.e. if there is an excess of $1 \mathrm{MW}$ generation that needs to be consumed by the cluster of controllable loads, there can be only one price that results in an extra consumption of $1 \mathrm{MW}$ by this cluster. This stable frequency dependent price is the optimal lagrange multiplier $\lambda_{f r e q}^{*}$.

The main advantage of the pricing method described in section III-C is that the devices don't need to have knowledge about system transfer functions. Besides that the customers don't need to do an iterative price update, but the price is directly linked to the measured frequency. Therefore the required local intelligence reduces. Finally analysing the stability is easy, as the aggregated demand behaves as a conventional generator.

\section{APPENDIX B \\ DAY-AHEAD PLANNING}

The day-ahead objective of the aggregator is to minimize its costs for providing the necessary energy:

$$
\min _{P_{t}^{D A}} \sum_{t=1}^{T} c_{t}^{D A} P_{t}^{D A} \triangle t
$$

where $c_{t}^{D A}$ is the expected day-ahead price at time step $t$. The amount of power that can be consumed each time step $P_{t}^{D A}$ is limited and can be defined based on historical information of daily flexibility bounds [16], [24]-[26]. The cluster limitations are:

$$
\begin{aligned}
P_{t}^{\min } & \leq P_{t}^{D A} \leq P_{t}^{\max } \\
E_{t+1} & =E_{t}+P_{t}^{D A} \triangle t \\
E_{t}^{\text {min }} & \leq E_{t} \leq E_{t}^{\max }
\end{aligned}
$$

If the aggregator has contracted reserves, these should be included in the cluster limitations.

$$
\begin{aligned}
& P_{t}^{\text {min }} \leq P_{t}^{D A}-R_{\text {contr }, t}^{1,+}-R_{\text {contr }, t}^{2,+} \\
& P_{t}^{D A}+R_{\text {contr }, t}^{1,-}+R_{\text {contr }, t}^{2,-} \leq P_{t}^{\text {max }} \\
& E_{t+1}^{+}=E_{t}^{+}+\left(P_{t}^{D A}-R_{\text {contr }, t}^{1,+}-R_{\text {contr }, t}^{2,+}\right) \triangle t \\
& E_{t+1}^{-}=E_{t}^{-}+\left(P_{t}^{D A}+R_{\text {contr }, t}^{1,-}+R_{\text {contr }, t}^{2,-}\right) \triangle t \\
& E_{t}^{\text {min }} \leq E_{t}^{+} \\
& E_{t}^{-} \leq E_{t}^{\text {max }}
\end{aligned}
$$

Similar to the extra constraints of section IV, the aggregator should limit the consumption in order to be able to provide the contracted reserves $R_{\text {contr }, t}^{1,2, \pm}$. Also, the aggregated cumulated energy of the cluster in case all upward or downward reserves would be activated is limited by the flexibility bounds of the cluster.
The required primary and secondary reserves are often guaranteed by long-term contracts in Europe [35], [36] and are therefore not considered to be variables in this work. The time of the day that reserves are contracted will have an impact on the profit of the aggregator, i.e. the minimum consumption to be able to provide upward reserves during peak periods can have a high cost. Future and ongoing work will focus on the day-ahead market decisions and the long-term contracts. The day-ahead planning can be further improved by taking into account the expected percentage of up or down regulation of each hour, which can be extracted from historical data [37].

\section{ACKNOWLEDGMENT}

The authors would like to thank B. Claessens and K. De Vos for the discussions on demand response systems.

\section{REFERENCES}

[1] A. Molina-Garcia, F. Bouffard, and D. Kirschen, "Decentralized demand-side contribution to primary frequency control," IEEE Trans. on Power Syst., vol. 26, no. 1, pp. 411 -419, feb. 2011.

[2] J. Short, D. Infield, and L. Freris, "Stabilization of grid frequency through dynamic demand control," IEEE Trans. on Power Syst., vol. 22, no. 3, pp. $1284-1293$, aug. 2007.

[3] J. Kondoh, N. Lu, and D. Hammerstrom, "An evaluation of the water heater load potential for providing regulation service," IEEE Trans. on Power Syst., vol. 26, no. 3, pp. 1309 -1316, aug. 2011.

[4] Z. Xu, J. Ostergaard, M. Togeby, and C. Marcus-Moller, "Design and modelling of thermostatically controlled loads as frequency controlled reserve," in Power Engineering Society General Meeting, 2007. IEEE, june 2007 , pp. $1-6$.

[5] J. Lopes, F. Soares, and P. Almeida, "Integration of electric vehicles in the electric power system," Proc. of the IEEE, vol. 99, no. 1, pp. 168 -183 , jan. 2011.

[6] H. Liu, Z. Hu, Y. Song, and J. Lin, "Decentralized vehicle-to-grid control for primary frequency regulation considering charging demands," IEEE Trans. on Power Syst., vol. 28, no. 3, pp. 3480-3489, Aug 2013.

[7] P. Almeida, J. Lopes, F. Soares, and L. Seca, "Electric vehicles participating in frequency control: Operating islanded systems with large penetration of renewable power sources," in PowerTech, 2011 IEEE Trondheim, june 2011, pp. $1-6$.

[8] Y. Rebours, D. Kirschen, M. Trotignon, and S. Rossignol, "A survey of frequency and voltage control ancillary services part i: Technical features," IEEE Trans. on Power Syst., vol. 22, no. 1, pp. 350-357, Feb 2007.

[9] B. Biegel, L. Hansen, P. Andersen, and J. Stoustrup, "Primary control by on/off demand-side devices," IEEE Trans. on Smart Grid, vol. PP, no. 99, pp. 1-11, 2013.

[10] S. Weckx, R. D’Hulst, and J. Driesen, "Optimal frequency support by dynamic demand." in PowerTech, 2013 IEEE Grenoble, 2013, pp. 1-6.

[11] P. Samadi, A. Mohsenian-Rad, R. Schober, V. W. S. Wong, and J. Jatskevich, "Optimal real-time pricing algorithm based on utility maximization for smart grid," in Proc. First IEEE Int Smart Grid Communications (SmartGridComm) Conf, 2010, pp. 415-420.

[12] P. Samadi, H. Mohsenian-Rad, R. Schober, and V. Wong, "Advanced demand side management for the future smart grid using mechanism design," IEEE Trans. Smart Grid, vol. 3, no. 3, pp. 1170-1180, 2012.

[13] P. Tarasak, "Optimal real-time pricing under load uncertainty based on utility maximization for smart grid," in Proc. IEEE Int Smart Grid Communications (SmartGridComm) Conf, 2011, pp. 321-326.

[14] K. De Craemer and G. Deconinck, "Balancing trade-offs in coordinated phev charging with continuous market-based control," IEEE PES Innovative Smart Grid Technologies 2012, 2012.

[15] M. D. Galus, R. Waraich, F. Noembrini, K. Steurs, G. Georges, K. Boulouchos, K. Axhausen, and G. Andersson, "Integrating power systems, transport systems and vehicle technology for electric mobility impact assessment and efficient control,' IEEE Trans. Smart Grid, vol. 3, no. 2, pp. 934-949, 2012.

[16] S. Vandael, B. Claessens, M. Hommelberg, T. Holvoet, and G. Deconinck, "A scalable three-step approach for demand side management of plug-in hybrid vehicles," IEEE Trans. on Smart Grid, vol. PP, no. 99, pp. $1-9,2012$. 
[17] K. Vanthournout, R. D'hulst, D. Geysen, and G. Jacobs, "A smart domestic hot water buffer," IEEE Trans. on Smart Grid,, vol. 3, no. 4, pp. $2121-2127$, dec. 2012.

[18] M. Fahrioglu and F. Alvarado, "Using utility information to calibrate customer demand management behavior models," IEEE Trans. on Power Syst.,, vol. 16, no. 2, pp. 317-322, 2001.

[19] M. Hommelberg, B. van der Velde, C. Warmer, I. Kamphuis, and J. Kok, "A novel architecture for real-time operation of multi-agent based coordination of demand and supply," in Proc. IEEE Power and Energy Society General Meeting - Conversion and Delivery of Electrical Energy in the 21st Century, 2008, pp. 1-5.

[20] K. Kok, B. Roossien, P. MacDougall, O. van Pruissen, G. Venekamp, R. Kamphuis, J. Laarakkers, and C. Warmer, "Dynamic pricing by scalable energy management systems ; field experiences and simulation results using powermatcher," in Power and Energy Society General Meeting, 2012 IEEE, july 2012, pp. $1-8$.

[21] D. P. Bertsekas, Constrained Optimization and Lagrange Multiplier Methods (Optimization and Neural Computation Series), 1st ed. Athena Scientific, 1996.

[22] C. Zhao, U. Topcu, and S. Low, "Optimal load control via frequency measurement and neighborhood area communication," IEEE Trans. on Power Syst., vol. PP, no. 99, pp. 1-12, 2013.

[23] P. Kundur, Power System Stability and Control, New York, 1994.

[24] K. De Craemer, S. Vandael, B. Claessens, and G. Deconinck, "An eventdriven dual coordination mechanism for demand side management of phevs," IEEE Trans. on Smart Grid,, vol. PP, no. 99, pp. 1-10, 2013.

[25] F. Ruelens, S. Vandael, W. Leterme, B. Claessens, M. Hommelberg, T. Holvoet, and R. Belmans, "Demand side management of electric vehicles with uncertainty on arrival and departure times," in Innovative Smart Grid Technologies (ISGT Europe), 2012 3rd IEEE PES International Conference and Exhibition on, 2012, pp. 1-8.

[26] B. Claessens, S. Vandael, F. Ruelens, and M. Hommelberg, "Selflearning demand side management for a heterogeneous cluster of devices with binary control actions," in Innovative Smart Grid Technologies (ISGT Europe), 2012 3rd IEEE PES International Conference and Exhibition on, 2012, pp. 1-8.

[27] J. Mathieu, M. Kamgarpour, J. Lygeros, and D. Callaway, "Energy arbitrage with thermostatically controlled loads," in Control Conference (ECC), 2013 European, 2013, pp. 2519-2526.

[28] Z. Chen, L. Wu, and Y. Fu, "Real-time price-based demand response management for residential appliances via stochastic optimization and robust optimization," IEEE Trans. on Smart Grid, vol. 3, no. 4, pp. 1822-1831, Dec 2012.

[29] A. Shapiro, D. Dentcheva, and A. Ruszczynski, Lectures on stochastic programming : modeling and theory, ser. MPS-SIAM series on optimization. Society for Industrial and Applied Mathematics, 2009.

[30] F. Ruelens, S. Weckx, W. Leterme, B. Claessens, S. Vandael, and R. Belmans, "Stochastic portfolio management of an electric vehicles aggregator,' in Innovative Smart Grid Technologies (ISGT Europe), 2012 4th IEEE PES International Conference and Exhibition on, 2013, pp. $1-8$.

[31] A. Conejo, J. Morales, and L. Baringo, "Real-time demand response model," IEEE Trans. on Smart Grid, vol. 1, no. 3, pp. 236-242, Dec 2010.

[32] K. De Brabandere, T. Loix, K. Engelen, B. Bolsens, J. Van Den Keybus, J. Driesen, and R. Belmans, "Design and operation of a phase-locked loop with kalman estimator-based filter for single-phase applications," in IEEE Industrial Electronics, IECON 2006 - 32nd Annual Conference on, Nov 2006, pp. 525-530.

[33] J. Van Roy, N. Leemput, S. De Breucker, F. Geth, P. Tant, and J. Driesen, "An Availability Analysis and Energy Consumption Model for a Flemish Fleet of Electric Vehicles," in European Electric Vehicle Congress (EEVC), Brussels, Belgium, October 26-28, 2011.
[34] E. Peeters, C. Develder, J. Das, J. Driesen, and R. Belmans, "Linear: towards a breakthrough of smart grids in Flanders," in Proc. 2nd Int. Conf. Innovation for Sustainable Production (i-SUP 2010), vol. 3, Bruges, Belgium, 18-21 Apr. 2010, pp. 3-6.

[35] K. D. Vos, "Sizing and allocation of operating reserves following wind power integration," Ph.D. dissertation, KULeuven, Leuven, 2013 [Online]. Available: http://www.esat.kuleuven.be/electa/publications/ fulltexts/pub_2446.pdf

[36] Y. Rebours, D. Kirschen, M. Trotignon, and S. Rossignol, "A survey of frequency and voltage control ancillary services mdash;part ii: Economic features," IEEE Trans. on Power Syst., vol. 22, no. 1, pp. 358-366, Feb 2007.

[37] E. Sortomme and M. El-Sharkawi, "Optimal charging strategies for unidirectional vehicle-to-grid," IEEE Trans. on Smart Grid, vol. 2, no. 1, pp. 131-138, March 2011.

\section{BIOGRAPHIES}

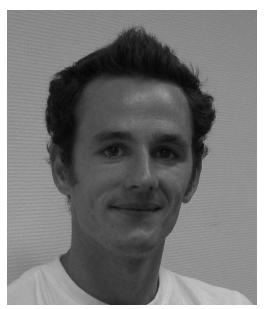

Sam Weckx (S'11) received the M.Sc. degree in Electrical Engineering in 2009 and Mechanical Engineering in 2010 from the Katholieke Universiteit Leuven (KU Leuven), Belgium, where he is currently working towards the Ph.D. degree as a research assistant with the division ESAT-ELECTA. His research interests include the application of distributed optimization in smart grids and voltage control in distribution networks.

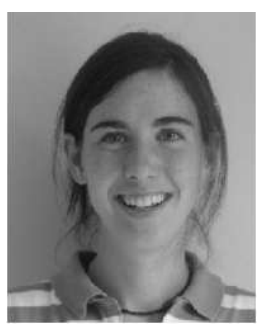

Reinhilde D'Hulst graduated in 2004 as M.Sc. in Electrical Engineering from the Katholieke Universiteit Leuven (KU Leuven). She obtained her Ph.D. in Electrical Engineering from the KU Leuven in 2009 after working on power management circuits for energy harvesters. Currently she works for VITO, the Flemish Institute for Technological Research where she is involved in several research projects related to Smart Grids. Currently she does research on grid-connecting issues of renewable energy resources and control algorithms for Demand Side

Management

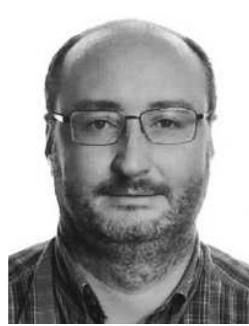

applications.
Johan Driesen (S'93 - M'97 - SM'12) received the M.Sc. and Ph.D. degrees in Electrical Engineering from the Katholieke Universiteit Leuven (KU Leuven), Leuven, Belgium, in 1996 and 2000, respectively. Currently, he is a Professor with the KU Leuven and teaches power electronics and electric drives. In 2000, he was with the Imperial College of Science, Technology and Medicine, London, U.K. In 2002, he was with the University of California, Berkeley. Currently, he conducts research on distributed generation, power electronics, and its 